eliminated (no matter how acid the urine might be), the pain subdued, and the temperature influenced, we need have no fear of heart-complication arising. Then comes the question, What are the best means, if there be any, to bring about this condition?

During the past three years, I have been in the habit of packing most of my cases in a wet blanket, and afterwards rolling them up in dry blankets, so as not only to promote profuse sweating, but also to increase the temperature. This mode of procedure, which I conducted in a very indefinite manner, gave such good results, that I thought carefully over the rationale of the system, and at once adopted a course of wet packing after the manner and with the success which I will relate to you. The procedure is simple. The bed is covered with Indiarubber sheeting; over this is laid a blanket which has been wrung out of hot water. The patient is then enveloped in the blanket, and covered with six folds of dry blanketing. By this, the temperature is raised and profuse sweating results : the former, if need be, is assisted by the administration of brandy in half-ounce or ounce doses every hour, and the latter by giving freely warm milk and water. If the temperature exceed 102 deg., then the stimulant is unnecessary. My plan is to continue the treatment for three successive days; namely, for six hours the first day, four the second, and two the third. After the first pack, the patient is free, or nearly so, from pain ; after the second pack, the pain has completely subsided, and after the third pack the sour smell usually disappears. In addition to the relief from pain and sub. sidence of acid secretions, the pyrexial state, with its attendant symptoms, will be found to decrease in direct ratio, and likewise the pulse. The secretion of urine will become more plentiful and the urea will diminish in quantity; yet, although the improvement is so marked in reference to pain, sweat, pulse, and temperature, the urine remains acid and loaded with lithates, and the tongue coated, for some days longer. It not unfrequently happens, especially in young people, when the weather is variable, that transitory pains return in one or more joints but in almost every instance the pain has been subdued, if not by the first, by the second bath. In reference to cardiac inflammation, I believe that this treatment subdues it more rapidly than any other, rendering the valves less likely to undergo organic change.

But now comes a very important practical question. There can be no doubt that the packing process produces considerable constitutional disturbance. Under what circumstances should this treatment be adopted, and under what conditions is it not practicable? Every one who has had much to do with thisdisease must be conscious of the anxiety which it gives him when the temperature exceeds I05 deg. or I06 deg., and especially when it is associated with the least sign of cerebral disturbance ; and, as my treatment consists in elevating the temperature, it will be apparent that some care is necessary. Thus, according to my experience, it should not be adopted-I. If the patient suffer from incompetency of the aortic valves; 2 . If there be much fluid in the pericardium from previous inflammation; 3 . If the temperature be over I04 deg.; 4. If the skin be hot, dry, and harsh, without the least tendency to sweating; 5. If there be extreme nervous prostration from habits of drunkenness or other vitiating cause ; 6 . If the patient be pregnant.

Again : during the time the patient is packed, the following points must be observed :-I. If, after two or three hours, the patient become very restless, with a dry non-perspirable skin, I should advise the treatment to be discontinued ; also when the temperature exceeds I05 deg.; 2. If the temperature do not rise, and the patient be sweating freely, give half an ounce, or even an ounce, of brandy every hour in warm milk and water. Thus we have to secure profuse sweating and a mean temperature of 104 deg.; we have to guard against a dry skin and a temperature over 105 deg.

Let us consider this a little more practically. If a healthy child be packed for six hours as directed, whose normal temperature is 99 deg., we find that it will only rise one degree during the whole course of the six hours, and the administration of a stimulant will not cause it to rise any more. It is very different during the pyrexial stage of acute rheumatism, and I have proved most unquestionably that, when the packing alone does not increase the temperature, this is easily brought about by giving brandy in the manner just stated. I have adopted this treatment with excellent results in cases where there has been, in addition to the rheumatic inflammation, a mitral murmur, pericarditis, and pleuropneumonia. In some cases, I give medicine, in others I do not. My rule is this : not to give medicine or solid food until after the third packing, and this means not until the acute symptoms have subsided and the temperature is down to roodeg.; then some vegetable tonic, with solution of acetate of ammonia, is to be preferred to large doses of alkali or quinine. If, however, the case have been of long cluration before coming under treatment, and if it be the third or fourth attack, with probably cardiac disease, then of course the orthodox measures must be resorted to for such complications. If the temperature should run very high, with tendency to delirium, I believe the best plan is in every instance, whether under the packing treatment or otherwise, to apply ice to the head, expose the body freely to a current of cold air, and sponge it over lightly with a mixture of one part of spirit to two of water, until the temperature falls ; then to discontinue this, and to apply a sinapism to the epigastrium. The following case occurred in my practice, from which a lesson might, perhaps, be learned. A young robust woman, aged I9, came under my care with incipient acute rheumatism, but with a dry harsh skin and a temperature of 102 deg. I had her packed in the usual manner at I.3O P.M.; at 4.30 P.M., there was no action of the skin; temperature, IO4 deg.; at 6.30 P.M., still no action of the skin; temperature 106.2 deg.; rapid action of the heart and tendency to delirium. I at once applied ice to the head, bathed the exposed body with spirit and water, and in twenty minutes I was pleased to find the temperature down to I03 deg.; the following morning it was ror deg. Again : a woman, aged 28, of drunken and dissolute habits, came under my care with the third attack of acute rheumatism ; there was considerable prostration and a temperature of 103.2 deg. I thought that I detected a fine murmur obscuring the second sound at the base of the heart. I ordered her to be packed in the usual way; but her temperature continued to increase, until it reached I Io deg., and she died. This is the only case which has terminated unfavourably out of a very large number. Whether the high temperature arose from the treatment, I am unable to say, and, as such cases do occur now and again under any treatment, I must decline to give an opinion.

\section{ON DIPHTHERIA.*}

By THOMAS PRANGLEY, M.R.C.S.Eng., Aylsham.

OUR acquaintance with diphtheria being of so recent a date as to render it doubtful whether we have as yet witnessed all its phases, is the excuse for bringing before you such facts and information as I was enabled to gather from notes of fifty-six cases which came under my notice during an outbreak which occurred in my neighbourhood in the summer and autumn of 1868 . As it is impossible in a paper of this kind to treat the subject in an exhaustive manner, I think I shall be best employing the time in discussing those points which may guide us in our prognosis and treatment; and shall make reference only to the cases which have fallen under my own observation, without availing myself of the labours of others.

During the epidemic which I witnessed, the disease assumed all degrees of severity; thus sometimes it so little affected the health, that the patient could remain at work, and would recover in a few days. In the majority of cases, however, the disease as a rule ran a much more severe course ; in some cases coming on so insidiously, that the patient was in the jaws of death before I saw him-killing not through any local complication, but by general poisoning of the system, characterised by the predominance of asthenic symptoms and the exhaustion of the vital powers. Other cases were characterised by symptoms of apnœa caused by the extension of the membrane to the nares, larynx, and trachea.

The actual "soreness" of the throat afforded no indication of the severity of the attack; for, though there was generally considerable pain during deglutition, in some cases it was so slight as to be scarcely appreciable. Thus I have notes of two cases of unusual severity, in which there was absolute immunity from pain on swallowing. In one of these, the throat was covered with a large grey membrane, and in the other case, that of a child, who ultimately died, both tonsils were covered with grey slough, and the lower part of the uvula sloughed; but there was no difficulty in swallowing. As a rule, it was more severe with children and in those cases which were ushered in with acute febrile symptoms, and was generally most experienced during the period of the membrane disappearing; so that I began to look upon the statement "that the medicine cut the throat" as a good sign; and the absence of all pain nearly always revealed on inspection a thick mass of grey slough, and was consequently a bad sign.

The variety of forms which the false membrane assumes, and the various conditions of the tonsils, are very remarkable; thus I have seen the membrane in consistence like glazed starch, cream, wet parchment, and a greyish flesh-like pulp of all degrees of colour, from the purest white to almost black. I have seen it in specks, patches, shreds, and in large firm membranes, forming an exact cast of the part it enveloped. I have seen the specks or patches surrounded by a bright red border,

* Read before the Medical Section at the Annual Meeting of the British Medical Association in Norwich, August 1874 . 
or gradually becoming thinner at the edges, imperceptibly losing itself, so that one could not exactly say how far it extended. I have seen the tonsils engorged to such an extent as to almost meet, or enlarged laterally, as if they had been flattened by a weight on their surfaces; and I have notes of one case in which the tonsils were rather depressed. The glands at the angle of the jaws were more or less enlarged; but I never saw them suppurate. In arranging my notes of various cases, I find that in some the membrane was confined to one tonsil throughoutbut these cases were rare ; in others, it affected both tonsils ; in others, the uvula was also affected, and in two cases sloughed off. In several, the membrane covered the palate and pharynx, and, in the most severe cases, spread into the nares and trachea.

The practical question is, Will these appearances in the throat guide us in the formation of a correct prognosis? I think this may be answered in the affirmative; for, if we do not find the tonsils engorged, or only enlarged laterally as if flattened, and if we find them covered only with specks or patches, and more especially if these be defined by a surrounding bright red border, and if the glands at the angles of the jaws be not much enlarged, we may foretell a favourable issue. If, on the other hand, we find the tonsils prominent, the fauces red or dusky, and if we find them covered with a glairy fluid like starch, or the patches undefined, so that we cannot satisfy ourselves where they terminate, we may be almost certain that the next time we examine we shall find the starch converted or the patches coalesced into one mass of thick membrane, and shall be more cautious in our opinion, and congratulate ourselves if our fears of a severe case be not realised.

The manner in which the membrane disappeared and the throat recovered varied considerably. In some cases, it broke up into shreds, and was either swallowed or expectorated with mucus ; in other cases, it exfoliated entire, and in these, similarly to what happens if it be artificially removed with the forceps, a new membrane appeared, but of less density than the one which preceded it. Thus I have seen as many as half-a-dozen successive membranes form one after the other, each one of less density than its predecessor, till at last a thin film of almost transparent lymph was the last trace of exudation. In other cases, the membrane retained its original form to the last, and seemed to imperceptibly waste day by day, becoming gradually thinner and more transparent; so that I frequently find such notes as these in my case-book : "Membrane seems to be gradually getting thinner"; "Membrane has gone apparently by absorption."

On a few occasions, after the entire disappearance of the membrane, I found the tonsils again speckled all over with small white cheesy spots. These need cause no alarm; for, though they remained in statu quo for some days, they always disappeared without further inconvenience.

One of the worst signs in this disease was the extension of the membrane to the nares, so that I looked suspiciuusly upon the unfortunate patient, who began to use the pocket-handkerchief too freely. This invasion of the nares was manifested by redness of the margin of the nostrils and a discharge of thin mucus, which rapidly became purulent and, as the disease progressed, very abundant; so that the sole occupation of the patient seemed to be wiping the nose. I possess notes of four cases in which this complication was very marked, and in neither was it delayed later than the sixth day. The constant discharge, acting as an irritant, soon produced excoriation of the edges of the nostril and upper lip ; and, in one severe case, these parts were covered with scabs, bleeding at the least touch. The spread to the nares may be apprehended when we find the membrane rapidly spreading over the soft palate; and, in all the cases I have seen, it appears to have reached the nares by this route, and not from an independent centre. In one case, the left nostril was blocked up with membrane, which was one day suddenly discharged, forming a complete cast of the nostril, and at once set the passage at liberty. While this nostril was blocked up, a very curious phenomenon occurred, showing at all events that the nasal duct was patent; whenever the patient swallowed fluids, some would come down the nose, and some would appear at the corner of the eye, making it smart. Of the cases in which the nasal complication was present, three died.

The propagation of diphtheritic membrane to the larynx and trachea is another ominous symptom. I have notes of seven cases-all children -in which this occurred, and of these four died. In some cases, it is the only dangerous complication, and may kill by asphyxiating the patient by suffocative paroxysms; and in others it is an additional burden to the unfortunate who has the disease already established in the nares. Its presence is usually first announced by a small dry cough of a peculiar character, resembling that of croup; then the voice becomes affected, and shortly the breathing becomes difficult, each inspiration producing a kind of whistling sound, As the disease progresses, the voice becomes more and more hoarse, till at last it is completely muffled, and the patient speaks in a whisper.

A peculiar feature which I have observed in these cases is an intermittence in the symptoms, which at first induced me to give a too favourable opinion, and to congratulate myself upon the efficacy of my treatment; thus I have seen the fits of dyspnœa reduced in frequency, the respiration becoming more regular, and an almost entire remission of laryngeal whistling; and then, at shorter and shorter intervals, especially at night, all would recur, the patient, apparently without any exciting cause, starting up in bed, making immense efforts to breathe, and, after a severe suffocative fit, falling back on the bed, blue in the face and exhausted. These fits may recur over and over again for some hours, and then another remission occurs, most probably only to be succeeded by a more severe exacerbation, till at last, after one severe effort, the agonies of death close the scene.

It may be well to pause here a moment to consider what is the cause of these intermissions, which so cruelly raise false hopes in the patient and his friends. Is it the displacement of a portion of the membrane in the trachea, being either coughed up, or, valve-like in its action, being pressed close to the walls by the force of inspiration, and thus permitting the free ingress of air? or does it resemble one of those pathological phenomena which we meet with, but cannot explain, in such diseases as cancer, where the pain is intermittent ? or does it, as in asthma, depend upon the additional element of spasm being added to the burdens? I think that the latter explanation is the most probable; for the suddenness of the attack, and its more frequent recurrence in the night, seem to favour this view.

In connection with this, I will call your attention to a phenomenon which frequently occurs in this disease, and that is "noisy breathing" during sleep. This is to be distinguished from laryngeal breathing, or we may unnecessarily alarm ourselves and patients. There is no stridor, no affection of the voice, and no suffocative paroxysms. It appears to me to arise from mere obstruction in the throat, and is still worse if the nares be implicated.

Time will only permit me to simply mention without comment certain sequelx. Of these, I have observed marked anæmia, dyspeptic symptoms, tendency to faintings, and various symptoms depending upon loss of nervous force, such as aphonia, paralysis of the palate, protracted difficulty in swallowing from paralysis of the pharynx, defective vision, depending upon loss of power in accommodation, such as presbyopia and diplopia, wry neck, and various degrees of general paralysis, from numbness and tingling of the hands and feet to total paralysis, preventing the patient from moving in any degree. Of these affections, paralysis of the soft palate and defective vision were the most frequent.

Passing over many interesting points in the clinical history of this disease, I proceed to the practical question of its treatment, which resolves itself into-local, directed to the throat itself; general, to combat with the great tendency to depression of the vital powers; and individual means to relieve certain symptoms which may arise during its course.

The local treatment I adopted in every case was the application of tincture of iodine (forty-eight grains to one ounce) to every part of the throat covered with membrane at least once in twenty-four hours, and the inhalation of iodine vapour mixed with steam, but more especially the latter, if the larynx were invaded. If the membrane were firm in texture, and not too strongly adherent, I always removed it and applied the tincture of iodine to the clenuded surface, and with the best results; for, although frequently the membrane would re-form, yet it never regained its pristine condition. If the membrane were in specks or shreds, I applied the iodine over them, and in general half-a-dozen applications were all that was required to procure their dismissal, and in several instances two applications were sufficient. This local application of iodine acts, not only as a caustic, but, I believe, confers a modifying influence upon the secreting structure, and further brings into action the power of the absorbents; thus tending to retard the spread of the membrane and to promote the removal of that which is formed. I am aware that, in urging this treatment, I am at issue with many who contend that, this disease being a general one depending upon certain changes in the blood by the introduction of a specific disease-poison, it is useless to attack the local manifestation of the disease any more than the pustules of smallpox. To those I would call attention to John Hunter's axiom, that two similar diseases cannot coexist in the system at the same time. For example, he states that, if you can succeed in changing the nature of an inflammation, you can often cure the original complaint. Higginbottom's method of arresting the spread of erysipelas by vesication with nitrate of silver is a familiar illustration of this Hunterian law ; and, if that treatment be effectual in erysipelas, why should not a 
similar treatment be equally efficacious in diphtheria? Again, I believe that the local manifestation of blood-poison is of itself a great indication for treatment ; for do not all poisons have some definite and specific action on some membrane or organ? Thus does not arsenic exert its influence chiefly on the mucous membrane of the stomach, colchicum on the ligaments, cantharides on the kidneys, and strychnine on the spinal cord? Then why should not the throat be the chief seat of the diphtheritic poison, and, if so, why should not the rational treatment be to arrest and destroy it at its first encampment? Again, if my experience be correct, that one of the most formidable symptoms we meet with is the extension of the membrane to the nares and trache, and, if my opinion be correct, that it does not arise there by an independent centre, but by extension of the membrane from the fauces, -is not this an additional reason for staying the progress of the membrane and limiting it to the fauces, whether we adopt caustics or any other means with that object in view? That iodine possesses this property, I feel convinced ; and, as I am not reading to you a treatise on diphtheria, but simply my own experience, I shall not enter into the merits or demerits of the various remedies which have been vaunted by numerous successive writers. The efficacy of any treatment is proved by its results; and, as I have stated, out of fifty-six cases which I have noted, seven died, of which five were moribund when seen, and only two died fairly after treatment.

The general treatment was supporting and stimulating throughout. A liberal supply of beef-ten, wine, and milk was frequently and regularly given, to maintain the system against the natural tendency to depression and exhaustion. In medicine, I rely upon chlorate of potash and tincture of steel, from three to five grains of the former with five to fifteen minims of the latter every four hours, according to age. When tracheal symptoms arise, I at once have recourse to the inhaler, beginning with ten drops, increasing to a drachm of the common tincture of iodine to a pint of boiling water, and letting the patient inhale as frequently as possible. In using this, one precaution is necessary, and that is not to begin with too large a supply of iodine, otherwise it is too irritating, causing the patient to cough, and making him unwilling to use it. I have found ten drops well borne to begin with; and, after a short time, we may gradually increase the quantity to a drachn to the pint without inconvenience. If this do good, which it undoubtedly does, it is evident it cannot be by any caustic action, but entirely through its modifying and absorbing influence upon the diseased tissue. I can refer to three cases in which this treatment was of marked utility. In cases where the fits of dyspnoea are severe and frequent, I have found nothing like an emetic of sulphate of copper, which generally expels a quantity of membrane from the larynx and trachea, and gives relief for a time at all events.

In tracheotomy I believe we may place considerable reliance, although my experience is limited to one case, and that unfortunately a fatal one ; yet I firmly believe that, if it be resorted to soon enough, we may rescue many lives. There is no doubt one feels inclined to put it off as long as possible ; for parents have a cur:ous repugnance to having their children's throats cut; and, if you are not successful, they speculate upon what assistance you afforded nature in her process of dissolution, and generally the balance is against the doctor; yet the evidence of numerous published cases, of which, roughly speaking, one-fourth were successful, proves that it is our duty not to neglect this chance of saving life, and more especially not to delay too long in resorting to it.

The treatment of the numerous sequelæ is difficult to formulate. I believe time and the vis medicatrix nature to be our most important agents; yet something can and ought to be done, for it would indeed be cruel to leave a poor patient totally paralysed without the hope of some assistance to the slow progress of nature. In those cases, I generally prescribe the liquor strychniae in five-minim doses, gradually increased to twelve, three times a day ; but I candidly confess I think it often did no good, for I well remember one case in which I gave it for a whole month, the paralysis steadily increasing the whole time. I then left it off, and prescribed a liniment as a placebo, when gradual improvement commenced, and recovery ultimately took place; but, on other occasions, I have found it decidedly of service, though I am doubtful whether it be more active than quinine, mineral acids, and other tonics. I believe we most show our discretion by changing our tonics, till we find the one best suited to the individual case, than by habitually using one formula indiscriminately. In addition to the tonic regimen, I recommend, when practicable, a judicious change of air and scene. On one occasion, I had resource to galvanism, with no satisfactory result. $I$ believe that rubbing and shampooing the limbs is of some service; at all events, it does no harm; and the simultaneous use of a little embrocation no doubt assists the charm. Some of the dyspeptic symptoms I found very troublesome to treat. As a rule, bismuth gave the most relief, combined with bland nourishment ; and, when the acute symptoms had subsided, a combination of quinine and strychnine was of marked benefit in giving tone to the digestive organs.

For the tendency to faintings which sometimes occurs, I have given a dose of ether ; but what is most important is to enjoin the recumbent posture till convalescence is well established, and even then I recommend a little wine to be taken before any exertion is used.

Having alluded to the general treatment of diphtheria and its sequels, there yet remains to be mentioned other minor points, which are of considerable importance to the suffering individual, by which we may alleviate suffering and hasten recovery. For example, where the patient complains of sore-throat $a b$ initio, I have found an iron gargle (two drachms of the tincture to half a pint of water) beneficial; but this I have found too cutting when the membrane has disappeared, and then $I$ have substituted one of borax and glycerine (two drachms of the former and one ounce of the latter to half a pint of water); and this gargle I have also found very useful to relieve the sensation of bread-crumbs and tickling in the throat, of which complaints are frequently made during convalescence. Where the denuded surface has proved to be ulcerated, a few touches of nitrate of silver assist the process of repair; and in one case where the tongue and roof of the mouth was so sore, that everything that was taken gave pain, I found that great relief was experienced by applying dry trisnitrate of bismuth powder with a camel's hair pencil just before taking food. In paralysis of the soft palate and regurgitation of fluids through the nostril, holding the nose during deglutition will be found very much to assist the act this a patient of mine found out for himself, and I subsequently con. firmed his statement in other cases. In these cases, too, I always advise thick drinks, which are more easily swallowed. When the pharynx is also affected, a large bolus is much more easily passed down than a small one, so that I have advised the collection of all the food in the mouth into one mass before swallowing. This is no doubt accounted for by the weakened muscular fibres of the pharynx being enabled to grasp a large bolus with less contractile effort, than would be required for a small one. In one case, I kept a patient alive for some days by giving the yelk of egg unbroken, and letting it glide down his throat, as it were. These are a few practical hints, which, from what I have seen, I believe to be useful; but the ingenuity of the practitioner will often be put to the test in coping with the anomalous symptoms which sometimes arise during the progress of this horrible, though interesting disease. Much might be written upon this important subject; in fact, a single case will often afford, in its chequered course, abundant matter for a long paper; but I have endeavoured to be as brief as possible, and, with that object in view, have entirely excluded the histories of cases which I should otherwise have liked to introduce.

\section{A CASE OF TUMOUR OF THE BRAIN, SIMU- LATING APOPLEXY.}

By ALFRED H. MARTIN, M.B.T.C.D., Assistant Medical Superintendent, Joint Counties' Asylum, Carmarthen.

M. L. was removed from Briton Ferry to this asylum in March 1868; and, from that period up to June 15 th, 1874 , had been under care and treatment in this institution. The assigned cause for her insanity was intemperance, and her aberration of intellect manifested itself by great irritability of temper, flying into ungovernable fits of passion without adequate provocation. The following extract from the case-book will show that the above history has been completely borne out.

"December 1873. Continues in fair bodily health and condition. Is employed regularly in the laundry, where she works steadily. Her mental state is much enfeebled; she is, however, coherent in her conversation, and replies to questions correctly. Is generally contented, and cheerful in manner and appearance; but, if thwarted in any way, becomes very much excited, loquacious, and abusive. Her desire for stimulants still exists, but is scarcely so prominent as formerly. Is always neat and clean in her personal appearance."

Since the above report was made, she had, in no material way, changed, up to the day of her death. She was occasionally troubled with slight attacks of dyspepsia, which yielded readily to appropriate reme. dies. She never made any special complaint of headache; there was no paralysis, either facial or in the extremities, and the pupils were perfectly equal and sensitive. Her sight, hearing, and other senses, were perfect.

On the evening of June 14 th, she complained of not feeling well, and, for the first time, of pain in her head. She appeared slightly excited, 